\title{
An Item Response Theory for Personality and Attitude Scales: Item Analysis Using Restricted Factor Analysis
}

\author{
David Thissen and Lynne Steinberg \\ University of Kansas
}

Thomas Pyszczynski University of Idaho

Jeff Greenberg lowa State University

This paper describes the applicability of item response theory to attitude scale development and provides an illustration derived from a study of the propensity toward jealousy in romantic relationships. The item analysis model used is identical to the factor analysis model, so factor analytic criteria are used to evaluate the scale. These criteria may be used to decide whether the scale may be scored as a measure of a single variable and whether a simple sum or a weighted sum of the item responses serves as an optimal test score. Estimates of the reliability of the scale based on the item response model are also described.

Personality and attitude measurement have been crucial in the historical development of psychology and continue to be cornerstones of empirical research. Considering the frequent use of various personality and attitude instruments in psychology, it is noteworthy that there has been little recent increase in the sophistication of the statistical techniques used in the construction of such scales. Variants of classical test theory are usually employed to evaluate the quality of an attitude or personality measure. In practice, the use of classical test theory in attitude scale construction frequently consists of piecemeal computation of the statistics of classical theory with little consideration of their meaning for the scale as a whole.

For instance, the "reliability" of the scale is usually computed using one of a number of historically interesting formulae. However, this statistic is often the only index of virtue reported for a newly constructed scale, regardless of the fact that a reliability of .8 may be obtained in any of the following ways: (1) a short test with items strongly related to the trait being measured, (2) a long test with either many mediocre items or some combination of good and poor items, or (3) a long test on which the items precisely measure two or three moderately related (or possibly unrelated) traits. The reliability of .8 under the conditions described in (1) represents good measurement; similar reliability obtained with (2) represents possibly acceptable measurement. However, such reliability obtained under the conditions described in (3) probably represents an instrument with little if any meaning.

To distinguish between (1) and (2), the classical theory offers the computation of item-score correlations, but to compute those correlations, the test must be scored. This poses a problem: Should the score be a simple sum or a weighted sum of the item responses or some transformation? It is difficult to decide before the item analysis is completed. In addition, item-score correlations do not distinguish (3) from (2); to do that requires factor analysis. If the scale is factor analyzed, however, should it be scored 
with raw scores or factor scores? What is the "reliability" of a factor score estimate? The answer to that question has been given by Cattell and Radcliffe (1962) and Heise and Bohrnstedt (1970), but the answer is not part of a system and so has not been widely used.

Item response theory (IRT) offers a superior alternative to the classical analysis. The essence of IRT is the specification of a statistical model for each item response in which the response is described as a function of the trait being measured and some item parameters. Since a statistical model is used, its parameters may be estimated and a statistical test of the fit of the model to the data may be computed. If the model is not rejected, then its consequences for item analysis and test scoring may be taken seriously. The parameters of the item response model may be used in item analysis and selection, and the form of the model may be used to specify the optimal test score (as an estimate of the value of the trait being measured) and its reliability, or standard error. If the model fits, the item parameters may also be used to score alternate forms of the scale, to handle missing data, and to equate forms. In contrast, classical test theory does not use a statistical model and therefore has no test of goodness of fit; it has only statistics whose applicability may be questioned.

Over the past 15 years, because of the shortcomings of classical test theory, IRT has been strongly advocated for the analysis of cognitive and ability tests (Bock \& Wood, 1971; Hambleton \& Cook, 1977; Lord, 1980; Weiss \& Davison, 1981). However, even though Damarin (1970) considered the problem some years ago, analysis of personality and attitude scales using IRT has lagged behind applications in ability measurement, probably because item response theorists have concentrated on models for binary (correct/incorrect) test items. Many attitude scales permit a broader range of response, such as those on a scale similar to those devised by Likert (1932), ranging from "strongly agree" to "strongly disagree." Thus, it has been erroneously believed that IRT is not applicable to attitude scales or that IRT was not applicable outside the domain of ability testing; there have been few applications of IRT to noncognitive measurement. One such study is Bejar's (1977) application to two personality scales of Samejima's (1973) model, which is for continuous responses of an unusual form: numbers chosen from the closed zero-one interval to reflect degrees of endorsement of the item. Andrich (1978) proposed a binomial model for Likert-style questionnaires which may be very useful for responses on 4- and 5-point scales. The bulk of the problem remains, however.

One of the most commonly used response forms for personality or attitude questionnaires is the 9point (or however-many-point) Likert-type scale, on which the respondent chooses some number on a relatively wide range to indicate some quantified response. Frequently, such scales are essentially unanalyzed from a test theoretic point of view; even classical theory is little used. The questions are invented with an eye toward face validity, the responses of each person are "added up," and an estimate of the reliability is computed using test-retest or internal consistency data. Common variations on this theme, such as the use of principal component scores, simply modify the "adding up" by including weights that maximize score variance. Such weights are unrelated to any statistical model and provide no certain improvement of the measurement.

Implicit in the usual scoring of attitude scales is the notion that the scale response for each item is linearly related to the variable being measured by the questionnaire. In the model described below this idea is used to develop an item response theory which states that an individual's response to an item on the questionnaire is a linear function of his/her trait value or attitude, plus or minus some random (Gaussian) error. This corresponds to traditional usage and provides an item response model from which the usual IRT routine follows.

\section{The Model}

Consider test-item response $x_{1 \mathrm{y}}$, which is the response of person $i$ to item $j$ where the response is a number on a continuous scale, such as a 9-point Likert-type scale. The following item response model 
has been (usually implicitly) used extensively in the analysis of such responses:

$$
\begin{array}{r}
x_{i j}=\mu_{j}+\lambda_{j} \theta_{i}+\varepsilon_{i j}, \\
\varepsilon_{i j} \sim N\left(0, \sigma_{j}\right)
\end{array}
$$

$\theta_{1}$, usually assumed to be scaled with mean zero and variance one, is the "latent" or "unobservable" individual differences variable (attitude, preference, personality trait, inclination) to be measured, and $\left\{\mu_{\jmath}, \lambda_{\jmath}, \sigma_{j}^{2}\right\}$ are the item parameters.

The model defined by Equation 1 is, of course, classical (one-factor) factor analysis (Lawley \& Maxwell, 1971). $\theta$, occupies the place of the "factor score" ( $\theta$ is used to emphasize the relationship of this theory with more current item response theory). The parameter $\mu_{\mathrm{J}}$ is the mean response to item $j$, and $\lambda_{1}$ is the "factor loading" for item $j$ (or the regression parameter for $x_{1}$ on $\theta_{1}$ ). The parameter $\sigma_{1}^{2}$ has traditionally been called the "unique variance" for item $j$. The model cannot be strictly true for 9-point Likert-type scale responses, due to discontinuity and end effects; but it may be approximately correct and has been widely used in practice. The Likert-type scale item response is considered to be a linear function of $\theta ; \lambda$ gives the slope, or expected change, in the scale response per unit change in $\theta$.

The problem of item parameter estimation for this model has been solved frequently over the past 50 years, but the discussion here will rest on the maximum likelihood procedures described by Lawley and Maxwell (1971) and Jöreskog (1970). The MLE for $\mu_{\jmath}$ is simply the mean item response; the MLEs for $\lambda_{1}$ and $\sigma_{1}^{2}$ are obtained with iterative computations making use of the variance-covariance matrix for $x_{13}$. The next section will make extensive use of estimates of $\lambda_{1}$ and $\sigma_{j}^{2}$ made subject to equality constraints. Under some such systems of constraints, as when all of the loadings are constrained to be equal, closed forms exist for the estimation of the parameters (Kristof, 1963, 1969). Jöreskog and his colleagues have developed versatile procedures for such restricted estimation in any case and have implemented those procedures in computer programs: first, RMLFA (Jöreskog \& Gruvaeus, 1967) and, more recently, COFAMM (Sörbom \& Jöreskog, 1976) and LISREL (Jöreskog \& Sörbom, 1978).

\section{Characterization of $\theta$ : Equal Weights}

After the parameters of $n$ items have been obtained by estimation using data from a suitably large sample ("item calibration"), they may be taken as fixed and known, and used in the measurement of people. Thus, if person $i$ takes the test, $\theta_{1}$ may be characterized by estimating the most likely location for $\hat{\theta}_{1}$ ( \pm its standard error) on the $\theta$ scale. The method of maximum likelihood provides a straightforward mechanism for this. Assuming that the responses are independent, conditional on $\theta$, the likelihood of the responses given by person $i, x_{1}$, is

$$
L\left(\underline{x}_{i} \mid \theta\right) \propto \Pi \exp \left[-\left(x_{i j}-\mu_{j}-\lambda_{j} \theta_{i}\right)^{2} / 2 \sigma_{j}^{2}\right]
$$

and the kernel of the loglikelihood is (suppressing indices of summation, which are always $j=1, n$, over items, in the sequel)

$$
\ell=-\Sigma\left(x_{i j}-\mu_{j}-\lambda_{j} \theta_{i}\right)^{2} / 2 \sigma^{2}
$$

$\hat{\theta}_{1}$ is the MLE when the partial derivative 


$$
{\frac{\partial \rho}{\partial \theta_{i}}}_{i}=\Sigma\left[\lambda_{j} / \sigma^{2}\right]\left(x_{i j}-\mu_{j}-\lambda_{j} \hat{\theta}_{j}\right)
$$

equals zero.

Now, if the $\lambda_{\mathrm{j}}$ are all equal and the $\sigma_{\mathrm{j}}^{2}$ are similarly all equal,

$$
\lambda_{j}=\lambda \text { and } \sigma_{j}^{2}=\sigma^{2} \text { for all } j
$$

Equation 2 is zero when

$$
\hat{\theta}_{i}=[1 / \lambda n]\left(\Sigma x_{i j}-\sum \mu_{j}\right)
$$

Thus, both the sum of the item responses $\left(\sum x_{1 \mathrm{y}}\right)$ and the average item response $\left(\sum \mathrm{x}_{1 /} / n\right)$ are proportional to $\hat{\theta}_{1}$, the MLE for the trait value in the item response model defined above. Both the sum and the average are widely used to score such scales; this result demonstrates that a sufficient condition for the sum/ average to be proportional to the MLE for $\theta$ is that a one-factor model with equality constraints is appropriate for the data. This condition is also sufficient, as Equation 4 is not zero for the estimate of Equation 6 unless all of the loadings (and unique variances) are equal.

\section{Characterization of $\theta$ : Unequal Weights}

If the equality constraints specified above are not met, there is still a closed form for the MLE $\hat{\theta}$, but it is a bit more complex. Under these circumstances, the MLE for $\hat{\theta}$ is

$$
\hat{\theta}_{i}=\left\{\Sigma\left[\lambda_{j} / \sigma^{2}\right]\left(x_{i j}-\mu_{j}\right)\right\} /\left[\sum\left(\lambda^{2} j / \sigma^{2}\right)\right]
$$

If it is assumed that the distribution of $\theta$ in the population is $N(0,1)$, then the loglikelihood is modified by the inclusion of an additional factor, so

$$
\ell *=-\sum\left(x_{i j}-\mu_{j}-\lambda_{j} \theta_{i}\right)^{2} / 2 \sigma^{2} j-\theta^{2} / 2
$$

and the resulting MLE $\hat{\theta}^{*}$ is

$$
\hat{\theta} *_{i}=\left\{\Sigma\left[\lambda_{j} / \sigma^{2}\right]\left(x_{i j}-\mu_{j}\right)\right\} /\left[\Sigma\left(\lambda^{2}{ }_{j} / \sigma^{2}{ }_{j}\right)+1\right]
$$

Note that Equations 9 and 7 are identical except for the "shrinkage factor" in the denominator. Also note that $\hat{\theta}_{\imath}^{*}$ is (ignoring location) a weighted average of the item responses, with the weights given by

$$
\beta_{j}=\left[\lambda_{j} / \sigma^{2}\right] /\left\{\Sigma\left(\lambda^{2}{ }_{j} / \sigma^{2}\right)+1\right\}
$$

These weights are identical to those developed by Ledermann (1939) for estimating factor scores, in a method called (by Harman, 1976, pp. 376 ff.) "estimation by theoretical model." Since the two estimates 
provided are linear transformations of each other, and location and scale are usually arbitrary in such scales in any event, it usually will not make any difference which form is used. This development simply serves to make explicit the logically identical status of "factor score estimates" in (one-factor) factor analysis and the "trait estimate" in contemporary categorical item response theory.

\section{Dimensionality and Application of the Model}

Three factor analytic outcomes are possible with Likert-type questionnaire data, and their consequences for the questionnaire-as-measurement are straightforward.

Condition l. Parameter estimation and measurement are both simplest under this condition; therefore, it may be considered a goal. This condition is met if a one-factor model could fit the observed data with the constraint that all of the item regressions on the common factor (factor loadings) are equal. Here, "fit" means that the standard likelihood ratio test does not demand rejection of the model. If this condition holds, the (unweighted) sum of the item responses for each person is a linear function of the maximum likelihood estimate of the attitude or trait value for that person. As a consequence the test is optimally scored by summing the individual item responses. This is what practitioners have routinely done. However, no proof of the necessity and sufficiency of this condition for the sum to be the maximum likelihood estimate is widely known; the results reported below will provide an illustration to illuminate the proof.

Condition 2. A one-factor model with unequal regression parameters provides a satisfactory fit to the observed test data. Under these circumstances the best estimate of the trait value (e.g., attitude) is a weighted sum of the item responses. The weights must be determined from the data, and that is difficult to do in a way which generalizes between samples.'

Condition 3. A one-factor model may not fit the observed test data. In this case there is statistical evidence that more than one source of variation among the individuals contributes to the item responses; the test seems to "measure more than one dimension." It is nearly impossible to score such a test so that the score represents a single conceptual entity.

With these conditions and their consequences specified, test construction for attitude scales is a relatively straightforward matter. A preliminary analysis of most unselected item pools will result in Condition 3. Experience with such data indicates that analysis of the residual correlations among the items (after "removal" of the first factor) should indicate any clusters of items which seem to be measuring other dimensions. Restricted factor analysis provides a mechanism to identify and remove these sets of items. They may be replaced if necessary to give a scale of the desired length. Eventually, removing items (and adding new ones) should give a scale which meets one of the first two conditions. Condition 1 is more desirable than Condition 2, because it is easier to score the test, so it may be worthwhile to eliminate items whose regressions deviate from the average for the test to obtain a set of items that meet Condition I. Data from a subsequent sample may be used to determine whether the revised scale still meets the statistical criterion.

The regression parameters under Conditions 1 and 2 may be used to compute the reliability of the test. Under Condition 1 the formula is simple: The estimated interitem correlation is $\hat{\lambda}^{2} /\left(\hat{\lambda}^{2}+\hat{\sigma}^{2}\right)$, and the classical Spearman-Brown formula may be used to extend that item reliability to the length of the test. Reliability gets higher as the test increases in length, but the test becomes more bothersome as it becomes longer. Information obtained in the item analysis may thus be used to allow the test constructor

\footnotetext{
'An unweighted sum may entail little loss (Laughlin, 1978; Wainer, 1976, 1978), but this matter needs further theoretical investigation in the context of factor score estimation.
} 
to create the shortest possible test that will measure exactly what is intended with a specified reliability. There are, therefore, three goals: (1) a single common factor model should fit the observed interitem covariance matrix, (2) the factor loadings should be equal, and (3) the length/reliability should be satisfactory.

\section{An Application of the Method}

The intent of the data analysis described here is test construction. To this end, somewhat ad hoc models will be used at times: models that include parameters or structure suggested by the data but not directly theoretically interpretable. This is not recommended practice for scientific model building. However, in this case, the ad hoc sections of the model will usually be directly related only to items which will eventually be abandoned due to their incomprehensible complexity with respect to the model under consideration. Use of a model that actually fits the data may reduce bias in the estimation of important structural parameters; therefore, data-directed model-modification will be used to achieve a satisfactory fit.

To clarify the consequences of these ideas, an illustration is provided below of scale construction making use of the techniques of restricted factor analysis. The topic of the attitude scale is romantic jealousy. Since substantive considerations are an important part of scale construction in this approach, a moderately detailed discussion of the subject matter is provided. However, the content of the scale is not important - the illustration is intended to be prototypic of attitude scale construction.

\section{The Subject of the Illustration:}

\section{Propensity Toward Jealousy in Romantic Relationships}

The work of White $(1979,1981)$ and Aronson and Pines (1979) indicates that there are individual differences in the propensity to experience jealousy. Variations in past experiences in relationships as well as experiences in a current relationship may affect the perception and interpretation of different situations. Therefore, the measurement instrument was comprised of a sample of situations described by short scenarios; the respondents evaluated the degree to which each situation (1) poses a threat to their relationship and (2) elicits jealousy. In this way, separate assessments may be made of the related concepts of threat and jealousy with respect to a relatively constant set of situations.

\section{Original Item Pool}

The questionnaire. The original item pool was intended to provide simultaneous measurement of jealousy and threat. Twenty items followed instructions which read

The following items provide brief descriptions of situations that might lead a person to feel jealous. Perhaps you have been in situations similar to those described. Think very carefully about how you would respond to such situations and rate the extent to which you would feel jealous in each situation. Your responses will be completely confidential and anonymous. Please try to be as accurate and honest as possible. Blanks (__ _ will refer to your partner in the relationship.

The first item was

1. You are at a party with W While you discuss your job with a close friend, s/he is on the opposite side of the room engaged in an intense discussion with an attractive member of the opposite sex. It appears that is greatly enjoying the discussion. 
A. To what extent would you see this situation as posing a threat to your relationship with

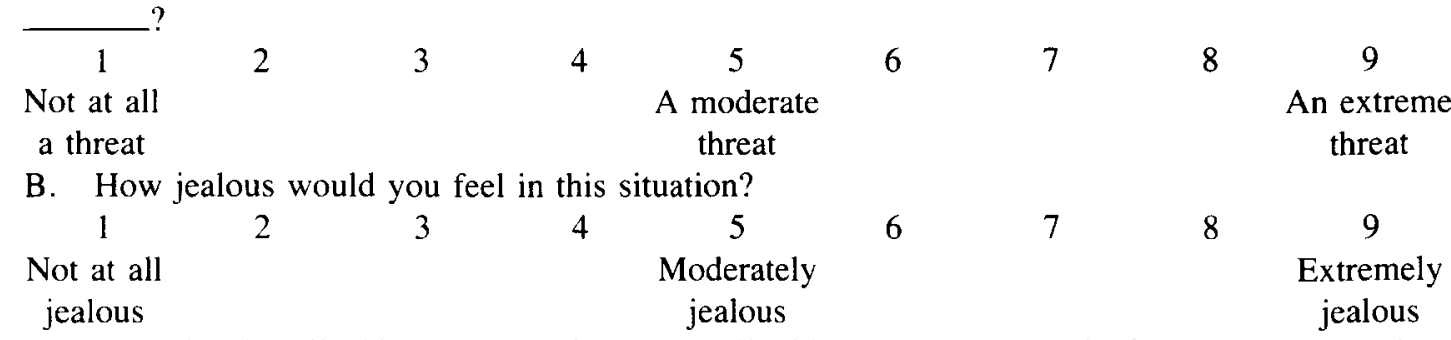
All of the scenarios described interactions of "_____ " with a third party, but the form of the interactions varied widely. The different scenarios included are listed in Table 1 . They were presented in the questionnaire in the order indicated by their item number but have been rearranged and grouped in Table 1 to clarify results to be discussed subsequently.

Subjects. The 20-item questionnaire was administered as part of a larger battery of personality instruments to 164 undergraduates at the University of Kansas. Of those providing complete data on the questionnaires, 79 indicated on a screening question that they were involved in a romantic relationship. Only the data for those 79 respondents are analyzed.

\section{Table 1}

Scenarios for the Original Item Pool

\begin{tabular}{|c|c|c|c|}
\hline$\overline{\text { Item }}$ & $\overline{\text { Set }}$ & Interaction with Third Party & Scale 2 \\
\hline 1 & & intense discussion at party & $\mathrm{JI}$ \\
\hline 2 & & take tennis lessions & $\mathrm{J} 2$ \\
\hline 4 & & describes as "sexy" & $\mathrm{T} 1$ \\
\hline 5 & & staring in restaurant & J6 \\
\hline 6 & & studying together & $\mathrm{T} 2$ \\
\hline 7 & A & receives expensive gifts & \\
\hline 10 & & goes on business trip & $\mathrm{J} 4$ \\
\hline 12 & & conversation at work & T3 \\
\hline 16 & & laughs at jokes & J5 \\
\hline 17 & & asks about third party & J3 \\
\hline 20 & & talks about classical music & T6 \\
\hline 18 & & is disturbed by rude remark & \\
\hline 3 & & together at restaurant & \\
\hline 8 & & leave party together & \\
\hline 9 & B & at bar together, touching & \\
\hline 14 & & reported sexual relations & \\
\hline 13 & & mutual friend "fun to be with" & \\
\hline 11 & & out to lunch with "old flame" & $\mathrm{T} 4$ \\
\hline 15 & $\mathrm{C}$ & spends time, "just good friends" & T5 \\
\hline 19 & & stranger "witty" & \\
\hline
\end{tabular}


Table 2

Covariance Matrix for the Original Item Pool (Lower Triangle) Residuals from the Single Factor Model for One Original Item Pool (Upper Triangle)

\begin{tabular}{|c|c|c|c|c|c|c|c|c|c|c|c|c|c|c|c|c|c|c|c|c|}
\hline Item & 1 & 2 & 4 & 5 & 6 & 7 & 10 & 12 & 16 & 17 & 20 & 18 & 3 & 8 & 9 & 14 & 13 & 11 & 15 & 19 \\
\hline 1 & 3.9 & .4 & .2 & .1 & .1 & -.2 &.$- \overline{3}$ & -.1 & .0 & .4 & -.1 & .1 & .5 & .1 & -.0 & -.6 & -.3 & -.3 & -.3 & -.1 \\
\hline 2 & 2.8 & 4.6 & -.2 & .4 & -.1 & -.1 & -.3 & -.4 & .1 & -.1 & -.2 & .1 & .8 & .3 & .1 & .1 & 0.1 & -.4 & -.5 & .0 \\
\hline 4 & 3.2 & 2.8 & 6.0 & .4 & .2 & -.0 & -.4 & .4 & .1 & .0 & -.4 & -.0 & -.7 & -.0 & .1 & .0 & .2 & 1 & .0 & -.3 \\
\hline 5 & 2.4 & 2.7 & 3.2 & 4.3 & -.0 & -.5 & -.5 & -.3 & .4 & -.2 & .1 & -.1 & .3 & -.1 & .1 & .3 & .1 & -.4 & -.3 & -.3 \\
\hline 6 & 2.9 & 2.7 & 3.7 & 2.7 & 5.7 & .3 & .2 & .1 & -.5 & -.4 & .2 & .0 & -.1 & -.1 & .1 & .2 & -.1 & .2 & .0 & .1 \\
\hline 7 & 1.8 & 2.0 & 2.5 & 1.4 & 2.7 & 5.5 & .6 & .7 & -.8 & .1 & -.9 & .5 & .4 & .9 & .3 & .3 & .0 & .0 & .4 & -.7 \\
\hline 10 & 2.6 & 2.6 & 3.1 & 2.2 & 3.6 & 3.1 & 6.1 & .8 & .1 & -.1 & .2 & -.2 & -.1 & $\overline{-.2}$ & -.2 & -.5 & .1 & .6 & .4 & -.0 \\
\hline 12 & 2.5 & 2.1 & 3.6 & 2.2 & 3.1 & 2.9 & 3.9 & $\overline{5.3}$ & .1 & .1 & -.2 & -.0 & $=.8$ & -.3 & -.4 & -.6 & .4 & .4 & .1 & -.3 \\
\hline 16 & 2.6 & 2.7 & 3.3 & 1.8 & 2.5 & 1.4 & 3.1 & 2.8 & 4.7 & .6 & .4 & -.0 & $\overline{-.3}$ & -.4 & -.5 & -.4 & .5 & $-\overline{-.2}$ & -.2 & -.1 \\
\hline 17 & 2.8 & 2.3 & 3.0 & 2.2 & 2.4 & 2.1 & 2.8 & 2.6 & 3.2 & 4.6 & .2 & .7 & -.1 & -.3 & -.7 & -.1 & .5 & -.3 & -.3 & -.0 \\
\hline 20 & 2.5 & 2.3 & 2.8 & 2.5 & 2.5 & 1.3 & 3.2 & 2.5 & 3.1 & 2.7 & 5.6 & .4 & .0 & -.4 & $\overline{-.0}$ & -.5 & .0 & -.4 & -.0 & .8 \\
\hline 18 & 1.0 & 0.9 & 1.1 & 0.7 & 1.1 & 1.2 & 0.8 & 0.9 & 0.9 & 1.6 & 1.3 & 3.6 & .0 & -.3 & -.4 & .2 & .0 & -.8 & -.4 & .1 \\
\hline 3 & 3.1 & 3.4 & 2.6 & 2.3 & 3.0 & 2.6 & 3.0 & 2.0 & 2.5 & 2.5 & 2.8 & 1.0 & 5.8 & 1.2 & .8 & .5 & -.8 & -.2 & -.0 & -.4 \\
\hline 8 & 2.0 & 2.2 & 2.3 & 1.7 & 2.1 & 2.5 & 2.1 & 1.7 & 1.6 & 1.6 & 1.6 & 0.4 & 3.2 & 3.9 & 1.4 & .8 & -.7 & .0 & -.3 & -.5 \\
\hline 9 & 2.1 & 2.3 & 2.8 & 2.2 & 2.6 & 2.1 & 2.4 & 1.9 & 1.8 & 1.5 & 2.3 & 0.4 & 3.1 & 3.1 & 4.9 & 1.5 & -.6 & -.0 & -.1 & -.2 \\
\hline 14 & 0.9 & 1.6 & 1.9 & 1.8 & 2.0 & 1.6 & 1.3 & 0.9 & 1.1 & 1.4 & 1.1 & 0.8 & 2.2 & 1.9 & 2.8 & 4.9 & -.4 & $\ldots 6$ & .2 & -.1 \\
\hline 13 & 1.5 & 1.7 & 2.4 & 1.9 & 2.0 & 1.6 & 2.3 & 2.3 & 2.4 & 2.3 & 1.9 & 0.6 & 1.2 & 0.8 & 1.1 & 0.8 & 3.0 & -.2 & .2 & .3 \\
\hline 11 & 2.2 & 2.2 & 3.2 & 2.1 & 3.2 & 2.2 & 3.7 & 3.1 & 2.5 & 2.2 & 2.3 & 0.1 & 2.5 & 2.1 & 2.3 & 2.2 & 1.7 & 5.6 & .5 & .8 \\
\hline 15 & 2.0 & 1.3 & 2.9 & 1.9 & 2.8 & 2.4 & 3.2 & 2.6 & 2.3 & 2.0 & 2.4 & 0.5 & 2.5 & 1.6 & 2.0 & 1.7 & 1.9 & 3.0 & 5.4 & .7 \\
\hline 19 & 2.5 & 2.6 & 3.0 & 2.4 & 3.2 & 1.6 & 3.1 & 2.5 & 2.7 & 2.6 & 3.6 & 1.1 & 2.5 & 1.6 & 2.2 & 1.5 & 2.2 & 3.6 & 3.3 & 5.1 \\
\hline
\end{tabular}

Note. Single underlines indicate large residual covariances between sequentially adjacent items; double underlines indicate other large residual covariances.

Results. The analysis began with the data from the "jealous" question only. Table 2 gives the variance-covariance matrix for the 9-point scale responses to the jealousy question for the 20 scenarios. If the jealousy question following all 20 scenarios measured a single underlying latent variable (that is, a propensity toward romantic jealousy), then the classical factor model with a single factor should fit the covariances in Table 2. It did not: The maximum likelihood approach to factor analysis provides a $G^{2}$ test (which follows a $\chi^{2}$ distribution) for the goodness of fit of the model, and the value obtained for a single factor model and the data in Table 2 was $G^{2}=343.4$ with 170 degrees of freedom, $p<.0001$. This indicates that the 20 items comprising the scale have more in common than a single underlying dimension. The scenarios cannot be used as an item pool without some selection.

Selection could proceed through arbitrary removal of poorly fitting items until the single-factor model fit; but such an approach, while widely used, is uninformative concerning the nature of the retained and excluded items. Informative selection must be based on further analysis of the data in Table 2 . A traditional approach to further analysis, given the lack of fit of a single factor, would be to attempt to fit the data with two unrestricted common factors. However, that approach is not particularly desirable here: It introduces the problem of rotation and ignores the fact that the theory implies that there should be a "general factor" among the responses, since they are all responses to the same question.

An exploratory procedure using the techniques of restricted factor analysis permits a more refined approach. The single common factor analysis described above provides an estimate of the covariance matrix; subtracting that from the observed covariance matrix gives a matrix of residuals, also shown in Table 2, which may be used to suggest improvements in the model. Examination of the residuals in Table 2 suggests three classes of large residuals from the single-factor model:

1. There are two "group factors," one for the group of items denoted "Set B" in Table 1 and another for "Set C"; Figure 1 provides a kind of schematic for the matrices of covariances and residuals, in which the two sets of residuals indicating group factors are in the regions corresponding to the areas labeled $\mathrm{BB}$ and $\mathrm{CC}$. 
2. There are also a number of large residual covariances between sequentially adjacent items; these are underlined in Table 2.

3. There are a few large residuals that are not covered by 1 and 2 ; these are double-underlined in Table 2.

The first two of these three classes of residuals are substantively interpretable, and all three classes can be incorporated in a restricted factor analysis model. Estimation of such a model and computation of its goodness of fit is possible using a variety of programs permitting constrained factor analysis; for the examples described here LISREL IV (Jöreskog \& Sörbom, 1978) was used. If such a model fits the data, the interpretation used to build that model may guide refinement of the item pool.

The residuals suggesting the two group factors are easily understandable. A distinction may be made between reactions of jealousy which arise from explicitly defined transgressions and those which arise from interpretations of ambiguous information. On the basis of that distinction, the ambiguous items of Set A (Table 1) contrast with the items of Set B (3,8,9, and 14) which explicitly or implicitly suggest sexual behavior, and one item (13) with negative residuals which euphemistically denies sexual involvement. This suggests a second factor, evaluation of sexual transgressions, with nonzero loadings only for the items of Group B and zero loadings elsewhere. Set C consists of three items which do not suggest sexual intimacy but rather suggest an explicit basis for the third-party interaction- " a belief in excuses" factor.

The large residuals between sequentially adjacent items suggest that for some pairs of stories, the response to the first story influenced the response to the second. This is not surprising, although it suggests lack of local independence. However, individual elements of the residual variance-covariance matrix may be estimated in addition to the common structure. This reduces the bias in the estimates of the common factor loadings which accrues from a poorly fitting model. Consequently, in the (now) three-factor model, estimated residual covariances were added corresponding to the underlined elements in Table 2. The seven double-underlined residual covariances in Table 2 are less easy to understand. There may be interactions in this set of scenarios which are not readily apparent, however, so estimated error covariances were added corresponding to those elements as well.

Estimation of the resulting restricted three-factor model (including 15 out of a possible 190 residual covariances) by maximum likelihood yielded a $G^{2}$ of 164.6 with 147 degrees of freedom, $p=.15$. The

\section{Figure 1}

A Schematic Representation of the Covariance Matrix for the Original Item Pool
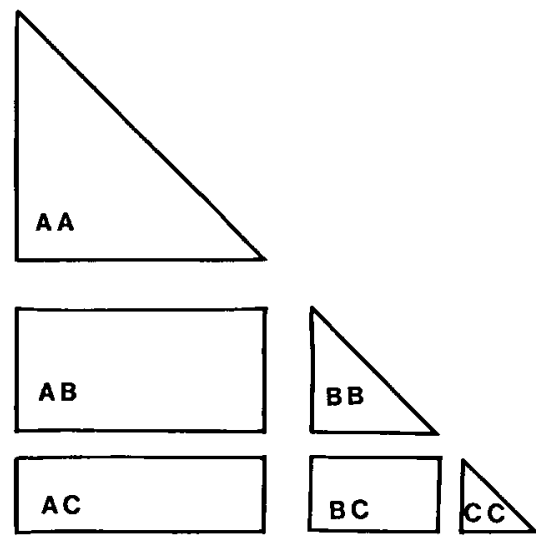
model is ad hoc; but that was intended, and it fits well enough. The estimated loadings are given in Table 3; all are many times their estimated standard errors, indicating that the estimates are reliable.

A parallel analysis performed on the items comprising the threat scale reveals that those data similarly could not be approximated by a single factor $\left(G^{2}=331.8\right.$ with 170 degrees of freedom, $\left.p<.0001\right)$. A complex model similar to the model for the jealousy question, but without the third factor, (barely) fit those data: $G^{2}=178.5$ with 149 degrees of freedom, $p=.05$.

To simplify the joint analysis of the jealousy and threat questions, only the scenarios of Set A (less Item 7, which did not fit very well) were retained for further analysis. Item 18 was omitted, as it was essentially unrelated to the remaining items. The variance-covariance matrix of the jealousy and threat questions for the reduced group of 10 items of Set A (less Item 7) is given in Table 4. Considering for the moment only the jealousy questions, a single factor model with equal loadings for all items, equal unique variances for all items, and "adjacent" residual covariances between three pairs of items provided an acceptable fit: $G^{2}=58.1$ with 50 degrees of freedom, $p=.2$. All five parameters had $t$ values (parameter/s.e.) over two. A similar model for the threat question alone, which differed only in permitting a higher loading for Items 1,4 , and 7 (and correspondingly lower unique variance) than for the other seven items gave marginal fit $\left(G^{2}=71.0\right.$ with 48 degrees of freedom, $\left.p=.02\right)$.

If all worked out as intended, an oblique two-factor model, with one factor (all loadings equal) for the jealousy questions, a second factor for the threat questions (with two distinct loadings), and appropriate error covariances between adjacent questions should fit the combined jealousy-threat matrix. Such a model

Table 3

Parameters and Standard Errors for the Three-factor, Correlated Residual Model for the Data in Table 2

\begin{tabular}{|c|c|c|c|c|c|c|c|c|}
\hline \multirow[b]{2}{*}{ Items } & \multicolumn{2}{|c|}{ Factor 1} & \multicolumn{2}{|c|}{ Factor 2} & \multicolumn{2}{|c|}{ Factor 3} & \multirow{2}{*}{$\begin{array}{l}\text { Unique } \\
\text { Variance }\end{array}$} & \multirow[b]{2}{*}{ S.E. } \\
\hline & Loading & S.E. & Loading & S.E. & Loading & S.E. & & \\
\hline 1 & 1.6 & .2 & & & & & 1.5 & .3 \\
\hline 2 & 1.5 & .2 & & & & & 2.3 & .4 \\
\hline 4 & 2.0 & .2 & & & & & 2.1 & .4 \\
\hline 5 & 1.5 & .2 & & & & & 2.0 & .3 \\
\hline 6 & 1.8 & .2 & & & & & 2.4 & .4 \\
\hline 7 & 1.3 & .2 & & & & & 3.8 & .6 \\
\hline 10 & 1.8 & .2 & & & & & 2.9 & .5 \\
\hline 12 & 1.6 & .2 & & & & & 2.5 & .4 \\
\hline 16 & 1.7 & .2 & & & & & 1.9 & .3 \\
\hline 17 & 1.6 & .2 & & & & & 2.1 & .4 \\
\hline 20 & 1.6 & .2 & & & & & 3.0 & .5 \\
\hline 18 & .6 & .2 & & & & & 3.2 & .5 \\
\hline 3 & 1.7 & .2 & 0.8 & .2 & & & 2.1 & .4 \\
\hline 8 & 1.2 & .2 & 1.2 & .2 & & & 1.0 & .3 \\
\hline 9 & 1.4 & .2 & 1.2 & .2 & & & 1.6 & .4 \\
\hline 14 & 0.9 & .2 & 0.7 & .2 & & & 3.4 & .6 \\
\hline 13 & 1.2 & .2 & -.6 & .2 & & & 1.2 & .2 \\
\hline 11 & 1.5 & .2 & & & 0.7 & .3 & 2.7 & .5 \\
\hline 15 & 1.5 & .2 & & & 0.6 & .3 & 2.9 & .5 \\
\hline 19 & 1.6 & .2 & & & 1.5 & .5 & 0.3 & 1.5 \\
\hline
\end{tabular}


was not even close; $G^{2}=778.1$ with 197 degrees of freedom, and the probability level is negligible. The large residuals causing this high value for the test statistic were between the jealousy and threat questions concerning the same story. When a single parameter was introduced corresponding to a residual covariance between questions within each scenario (all such covariances assumed equal), the $G^{2}$ dropped to 295.3 , a reduction of 482.8 with a single degree of freedom. The loading matrix for this model is given in Table 5 ; the factors are correlated $.8($ s.e. $=.05)$.

\section{Discussion: Original Item Pool and Sample I}

It is not clear that more would be gained by further analysis of the complex results from the first set of data. The final model did not fit; but it is not necessary to find an entirely satisfactory model for a questionnaire which is to be modified immediately. Changes are required in the scale, but what changes? Or from this exploratory analysis (the image conveyed by the restricted maximum likelihood and the $p$ values notwithstanding, since this has been exploratory analysis), what has been found? There are three answers:

1. The subject matter of the scenarios used to stimulate jealousy and threat responses should be ambiguous; it seems that the "single general factor" may be a tendency to interpret these scenarios in a way evoking jealousy or threat, and ambiguity is necessary to allow such various interpretations. Explicit scenarios elicited complex, seemingly multidimensional responses.

2. Adjacency of some of the stories spuriously increased their intercorrelation; some stories are not so useful immediately following others.

3. The procedure of including both jealousy and threat questions following each scenario led to responses that are more highly correlated within scenarios than were the jealousy and threat responses between scenarios; the latter presumably measures the true correlation of jealousy and threat. As this questionnaire is being developed for research concerning the relationship of jealousy and threat with other social/personality variables, separate questionnaires will be required.

\section{Revised Questionnaire}

Items and subjects. Revised jealousy and threat scales were constructed using the information developed from the analysis of the first set of data. Changes were made which were intended to bring the new scales closer to the psychometric goals: acceptance of a unifactorial equal weight regression model, and minimum length compatible with a reliability between .8 and .9 .

The estimated correlations between the "good" items (Set A) of the original pool averaged about .5. If such an interitem correlation is assumed for the finished scale, then the well known SpearmanBrown formula (see Lord \& Novick, 1968, pp. 112-114) gives a reliability of .86 for a six-item test: this is in the middle of the target range, so a test length of six items was chosen. To reduce problems caused by "error correlations" between the threat and jealousy questions following the same scenario, two separate questionnaires were constructed-one to measure threat and the other for jealousy. The two questionnaires made use of mutually exclusive sets of the story topics taken from the original item pool: the stories making up the revised jealousy questionnaire are marked $\mathrm{J} 1-\mathrm{J} 6$ in Table 1 , and the threat questionnaire used the stories marked $\mathrm{T} 1-\mathrm{T} 6 .^{2}$

'Item 7 was omitted from the revised scales due to large residual covariance with several of the other items. Items 11 and 15 were included with those of Set $\mathrm{A}$ in the revised threat scale. since there was no evidence of the third factor for Set $\mathrm{C}$ in the original threat data. 
Table 4

Jealousy and Threat Reduced Original Item Sets, 10 Items Each

\begin{tabular}{|c|c|c|c|c|c|c|c|c|c|c|c|c|c|c|c|c|c|c|c|c|}
\hline Item & $\mathrm{J} 1$ & $\mathrm{Tl}$ & $\mathrm{J} 2$ & T2 & $\mathrm{J} 3$ & T3 & .54 & T4 & $\mathrm{J} 5$ & T5 & $\mathrm{J} 6$ & $\mathrm{~T} 6$ & $\mathrm{~J} 7$ & $\mathrm{~T} 7$ & $\mathrm{~J} 8$ & $\mathrm{~T} 8$ & J9 & T9 & $\mathrm{J} 10$ & $\overrightarrow{\mathrm{T} 10}$ \\
\hline $\mathrm{J1}$ & $3 . \overline{9}$ & & & & & & & & & & & & & & & & & & & \\
\hline $\mathrm{T} 1$ & 2.6 & 3.1 & & & & & & & & & & & & & & & & & & \\
\hline $\mathrm{J} 2$ & 2.8 & 1.9 & 4.6 & & & & & & & & & & & & & & & & & \\
\hline $\mathrm{T} 2$ & 2.3 & 2.5 & 3.5 & 4.7 & & & & & & & & & & & & & & & & \\
\hline J3 & 3.2 & 2.2 & 2.8 & 2.0 & 6.0 & & & & & & & & & & & & & & & \\
\hline $\mathrm{T} 3$ & 2.7 & 2.9 & 2.4 & 3.0 & 4.1 & 5.0 & & & & & & & & & & & & & & \\
\hline J4 & 2.4 & 1.3 & 2.7 & 1.9 & 3.2 & 2.4 & 4.3 & & & & & & & & & & & & & \\
\hline T4 & 1.6 & 1.6 & 2.0 & 2.1 & 2.1 & 2.4 & 2.8 & 3.2 & & & & & & & & & & & & \\
\hline J5 & 2.9 & 2.0 & 2.7 & 1.5 & 3.7 & 2.5 & 2.7 & 1.6 & 5.7 & & & & & & & & & & & \\
\hline T5 & 2.3 & 2.3 & 2.3 & 2.3 & 2.6 & 3.0 & 1.7 & 1.5 & 3.7 & 4.7 & & & & & & & & & & \\
\hline J6 & 2.6 & 1.4 & 2.6 & 1.1 & 3.1 & 1.6 & 2.2 & 1.1 & 3.6 & 2.2 & 6.1 & & & & & & & & & \\
\hline T6 & 2.0 & 2.1 & 1.6 & 1.8 & 2.1 & 2.3 & 1.1 & 1.3 & 2.4 & 2.4 & 3.8 & 4.5 & & & & & & & & \\
\hline J7 & 2.5 & 1.2 & 2.1 & 1.0 & 3.6 & 1.9 & 2.2 & 0.8 & 3.1 & 2.1 & 3.9 & 2.5 & 5.3 & & & & & & & \\
\hline T7 & 1.9 & 1.6 & 1.4 & 1.5 & 2.7 & 2.5 & 1.5 & 1.1 & 1.9 & 2.1 & 2.2 & 2.5 & 3.8 & 4.0 & & & & & & \\
\hline $\mathrm{J} 8$ & 2.6 & 1.7 & 2.7 & 2.3 & 3.3 & 2.7 & 2.8 & 2.2 & 2.5 & 2.1 & 3.1 & 2.3 & 2.8 & 2.2 & 4.7 & & & & & \\
\hline$T 8$ & 1.6 & 1.8 & 1.8 & 2.6 & 1.8 & 2.5 & 1.7 & 1.8 & 1.1 & 1.8 & 1.5 & 2.0 & 1.6 & 2.0 & 3.1 & 3.7 & & & & \\
\hline J9 & 2.8 & 2.0 & 2.3 & 1.9 & 3.0 & 2.5 & 2.2 & 1.3 & 2.4 & 2.4 & 2.8 & 2.1 & 2.6 & 1.8 & 3.2 & 2.3 & 4.6 & & & \\
\hline T9 & 2.5 & 2.6 & 1.8 & 2.4 & 2.4 & 3.2 & 1.6 & 1.9 & 1.9 & 2.5 & 2.0 & 2.5 & 1.7 & 2.2 & 2.8 & 2.8 & 3.4 & 4.1 & & \\
\hline J10 & 2.5 & 1.5 & 2.3 & 1.5 & 2.8 & 2.2 & 2.5 & 1.8 & 3.2 & 2.2 & 3.2 & 1.9 & 2.5 & 1.9 & 3.1 & 1.6 & 2.7 & 2.3 & 5.6 & \\
\hline T10 & 2.0 & 1.8 & 1.8 & 1.9 & 2.1 & 2.3 & 1.5 & 1.5 & 2.0 & 2.3 & 2.3 & 2.5 & 1.8 & 2.0 & 2.3 & 1.9 & 2.2 & 2.7 & 3.7 & 4.5 \\
\hline
\end{tabular}

Table 5

Parameters of the Two-Factor Mode1 Original Samples Reduced Item Set

\begin{tabular}{|c|c|c|c|c|}
\hline Item & $\begin{array}{l}\text { Factor } \\
\text { Loading }\end{array}$ & $\begin{array}{l}1 \\
\text { S.E. }\end{array}$ & $\begin{array}{l}\text { Factor } \\
\text { Loading }\end{array}$ & S.E. \\
\hline $\mathrm{J} 1$ & 1.7 & .1 & & \\
\hline $\mathrm{T} 1$ & & & 1.6 & .1 \\
\hline $\mathrm{J} 2$ & 1.7 & .1 & & \\
\hline $\mathrm{T} 2$ & & & 1.4 & .1 \\
\hline J3 & 1.7 & .1 & & \\
\hline T3 & & & 1.6 & .1 \\
\hline J4 & 1.7 & .1 & & \\
\hline T4 & & & 1.4 & .1 \\
\hline J5 & 1.7 & .1 & & \\
\hline T5 & & & 1.4 & .1 \\
\hline J6 & 1.7 & .1 & & \\
\hline $\mathrm{T} 6$ & & & 1.4 & .1 \\
\hline J7 & 1.7 & .1 & & \\
\hline T7 & & & 1.4 & .1 \\
\hline J8 & 1.7 & .1 & & \\
\hline T8 & & & 1.4 & .1 \\
\hline J9 & 1.7 & .1 & & \\
\hline T9 & & & 1.6 & .1 \\
\hline $\mathrm{J} 10$ & 1.7 & .1 & & \\
\hline $\mathrm{T} 10$ & & & 1.4 & .1 \\
\hline
\end{tabular}


The revised jealousy questionnaire was formally very similar to the original item pool; the instructions and the first question follow.

Instructions: The following items provide brief descriptions of situations that might lead a person to feel jealous. Perhaps you have been in situations similar to those described. Think very carefully about how you would respond to such situations and rate the extent to which you would feel jealous in each situation. Please try to be as accurate as possible. Blanks (__ will refer to your partner in the relationship; when you encounter a blank, mentally fill it in with the name of your partner. 1. You are at a party with W While you discuss your job with a close friend, s/he is on the opposite side of the room engaged in an intense discussion with an attractive member of the opposite sex. It appears that__ is greatly enjoying the discussion.

How jealous would you feel in this situation?

$\begin{array}{ccccccccc}1 & 2 & 3 & 4 & \begin{array}{c}5 \\ \text { Moderately } \\ \text { jealous } \\ \text { jealous }\end{array} & & \begin{array}{c}\text { jeall } \\ \text { jealous }\end{array} & & \end{array}$

In contrast, the revised threat questionnaire was somewhat different from the original. To emphasize the objective nature of the threat question, the instructions were different, and the scenarios were stated entirely in the third person. There were no blanks into which the respondent was to project the name of "your partner." There were two forms, one for males and one for females, to preserve appropriate (heterosexual) gender relationships between the names in the stories and the respondents. The instructions and the first question of the revised threat questionnaire follow; the complementary material from the version for females is indicated in parentheses.

Instructions: The following items provide brief descriptions of the situations one may encounter in a relationship. Please read through these situations and evaluate the extent to which the event would present a threat to the relationship; that is, could lead to a possible break-up of the relationship.

1. Sue (Tim) is involved in a romantic relationship with Tim (Sue). Sue (Tim) and Tim (Sue) have a mutual friend, Bob (Judy), and the three of them have been friends for some time. Sue (Tim) confides in Tim (Sue) that she (he) thinks Bob (Judy) is extremely sexy.

To what extent would you see this situation as posing a threat to Sue and Tim's relationship?

Not at all

$2-3=4$

A moderate

threat

An extreme

a threat

These two revised questionnaires were administered to a new sample of 141 University of Kansas
72 indicated on the screening question that they were currently involved in a undergraduates, of whom 72 indicated on the screening question that they were currently involved in a romantic relationship.

Results. Both scales met the specified criteria. Estimation of the parameters of a one-factor model with equal loadings for the jealousy scale only (the bottom-right $6 \times 6$ covariance matrix in Table 6) gave $\hat{\hat{\lambda}}=1.4$ (s.e. $=.1$ ), $\hat{\sigma}^{2}=2.0$ (s.e. $=.1$ ), and a goodness of fit $G^{2}=14.0$ with 19 degrees of freedom, $p=.8$. The estimated interitem correlation was $1.4^{2} /\left(1.4^{2}+2.0\right)=.5$, which gives the expected Spearman-Brown reliability for the scale of .86 .

Similar estimation for the threat questionnaire gave $\hat{\lambda}=1.2($ s.e. $=.1), \hat{\sigma}^{2}=2.0($ s.e. $=.2)$, and a goodness of fit $\mathrm{G}^{2}$ of 19.3 with 19 degrees of freedom, $p=.4$. The estimated interitem correlation was $1.2^{2} /\left(1.2^{2}+2.0\right)=.4$, which gives a Spearman-Brown reliability for the sum of the six items of .80 .

A further test of the model involved simultaneous estimation of the parameters of a restricted twofactor model for the threat and jealousy data in Table 6. The first factor was permitted to have nonzero, equal loadings for the threat items only; the second factor, for the jealousy items only. The unique 
Table 6

Variance-Covariance Matrix for the Revised Scales in the Second Sample

\begin{tabular}{|c|c|c|c|c|c|c|c|c|c|c|c|}
\hline \multicolumn{6}{|c|}{ Threat } & \multicolumn{6}{|c|}{ Jealousy } \\
\hline 1 & 2 & 3 & 4 & 5 & 6 & 1 & 2 & 3 & 4 & 5 & 6 \\
\hline 3.7 & & & & & & & & & & & \\
\hline 1.6 & 3.0 & & & & & & & & & & \\
\hline 1.0 & 1.2 & 2.4 & & & & & & & & & \\
\hline 1.3 & 1.6 & 1.4 & 3.8 & & & & & & & & \\
\hline 1.8 & 1.8 & 0.8 & 1.7 & 3.6 & & & & & & & \\
\hline 1.2 & 1.4 & 0.9 & 1.2 & 1.6 & 3.7 & & & & & & \\
\hline 1.7 & 1.7 & 0.7 & 1.4 & 1.2 & 1.2 & 4.0 & & & & & \\
\hline 1.4 & 1.8 & 1.1 & 1.7 & 1.7 & 1.2 & 2.2 & 3.7 & & & & \\
\hline 1.8 & 1.4 & 1.1 & 0.9 & 1.2 & 1.3 & 2.5 & 2.0 & 4.4 & & & \\
\hline 1.0 & 0.9 & 0.9 & 1.3 & 0.9 & 0.5 & 1.8 & 1.3 & 1.9 & 3.5 & & \\
\hline 1.5 & 1.3 & 1.0 & 1.0 & 1.2 & 1.2 & 2.0 & 1.9 & 1.9 & 1.5 & 3.5 & \\
\hline 1.5 & 1.7 & 1.0 & 1.2 & 1.8 & 1.1 & 2.3 & 2.2 & 2.5 & 1.3 & 2.1 & 4.7 \\
\hline
\end{tabular}

variances were constrained to be equal within the two scales, and the two factors were permitted to be correlated (oblique). The estimates are in Table 7; the model fit, $\mathrm{G}^{2}=65.1$ with 73 degrees of freedom, $p=.7$. The estimated correlation between the traits underlying threat and jealousy was .77 (s.e. $=.08$ ). That suggests the hypothesis that there may be only one factor here-equivalent to the hypothesis that the correlation between these two factors is 1.0. That hypothesis was tested by estimating the parameters of the model with that correlation fixed at $1.0 ; G^{2}$ was 86.7 , giving a decrease-in- $G^{2}$ test of the difference between the correlation in question (.77) and 1.0 of $86.7-65.1=21.6$ with 1 degree of freedom, $p<.0001$. This test is the multivariate extension of the test of the hypothesis that two variables measure the same trait, developed by Lord (1957). In this case, the hypothesis that only a single factor is being measured, not two, was rejected.

Discussion. It is somewhat surprising that this test construction procedure was successful in only two "iterations." One would expect to learn, again, from Sample II and to go on to Sample III (or perhaps IV) to develop the scale that met the criteria. It may be that jealousy and threat are easy to measure. It is likely, however, that the existence of the very precise criteria for scale construction used here--one factor, equal loadings, specified reliability-facilitate fast scale construction.

\section{Conclusions}

Within the framework of item response theory, this paper has developed a set of criteria for attitude scale construction, and has described and illustrated a set of procedures to meet those criteria; these procedures and criteria should greatly simplify the construction of multi-item scales to measure specific attitudes or traits.

Straightforward goals of scale construction yield the criteria. A scale should (1) measure one attitude or trait; (2) be optimally scored by simple summation of the item responses; and (3) be as short as possible, compatible with its desired reliability. Those goals, in combination with a suitable item response model, yield the criterion that the fit of a single-common-factor model with equal item loadings should not be rejected, using the standard likelihood ratio test and a reasonable sample size. The sample does 
Table 7

Parameters and Standard Errors of Equal Weights Models for the Data of Table 6

\begin{tabular}{|c|c|c|c|c|c|c|}
\hline \multirow[b]{2}{*}{ Item } & \multicolumn{2}{|c|}{ Factor 1} & \multicolumn{2}{|c|}{ Factor 2} & \multicolumn{2}{|l|}{ Unique } \\
\hline & Loading & S.E. & Loading & S.E. & Variance & S.E. \\
\hline $\mathrm{T} 1$ & 1.2 & .1 & & & 2.01 & .15 \\
\hline $\mathrm{T} 2$ & 1.2 & .1 & & & 2.01 & .15 \\
\hline T3 & 1.2 & .1 & & & 2.01 & .15 \\
\hline T4 & 1.2 & .1 & & & 2.01 & .15 \\
\hline T5 & 1.2 & .1 & & & 2.01 & .15 \\
\hline T6 & 1.2 & .1 & & & 2.01 & .15 \\
\hline $\mathrm{J} 1$ & & & 1.4 & .1 & 1.98 & .15 \\
\hline $\mathrm{J} 2$ & & & 1.4 & .1 & 1.98 & .15 \\
\hline $\mathrm{J} 3$ & & & 1.4 & .1 & 1.98 & .15 \\
\hline $\mathrm{J} 4$ & & & 1.4 & .1 & 1.93 & .15 \\
\hline $\mathrm{J} 5$ & & & 1.4 & .1 & 1.93 & .15 \\
\hline J6 & & & 1.4 & .1 & 1.98 & .15 \\
\hline
\end{tabular}

not need to be very large, since only two parameters are estimated: the common loading and the unique item variance. The estimated interitem correlation is then easily computed, and the Spearman-Brown formula gives the length of the final scale and/or its reliability.

The computations necessary for all of this make use of widely available computer programs for constrained estimation in the factor analysis model. In this paper LISREL IV (Jöreskog \& Sörbom, 1978) has been used; other programs may be available now or in the near future. If the criteria specified here were met for attitude scales constructed in the future, use and interpretation of those scales would be greatly enhanced.

\section{References}

Andrich, D. A binomial model for the study of Likertstyle questionnaires. British Journal of Mathematical and Statistical Psychology, 1978, 31, 84-98.

Aronson, E., \& Pines, A. Exploring sexual jealousy. Unpublished manuscript, 1979.

Bejar, I. An application of the continuous response level model to personality measurement. Applied Psychological Measurement, 1977, 1, 509-521.

Bock, R. D., \& Wood, R. Test theory. Annual Review of Psychology, 1971, 22, 193-224.

Bohm, E. Jealousy. In A. Ellis \& A. Abarbanel (Eds.), The encyclopedia of sexual behavior (Vol. 1). New York: Hawthorn, 1961.

Cattell, R. B., \& Radcliffe, J. Reliability and validity of simple and extended weighted and buffered unifactor scales. British Journal of Statistical Psychology, 1962, 15, 113-128.
Damarin, F. A latent structure model for answering personal questions. Psychological Bulletin, 1970, 73, 2340 .

Hambleton, R. K., \& Cook, L. L. Latent trait models and their use in the analysis of educational test data. Journal of Educational Measurement, 1977, 14, 7595.

Harman, H. H. Modern factor analysis. Chicago: University of Chicago Press, 1976.

Heise, D. R., \& Bohrnstedt, G. W. Validity, invalidity, and reliability. In E. F. Borgatta \& G. W. Bohrnstedt (Eds.), Sociological methodology 1970. San Francisco: Jossey-Bass, 1970.

Hull, C. H., \& Nie, N. H. SPSS Update 7-9. New York: McGraw-Hill, 1981.

Jöreskog, K. G. A general method for the analysis of covariance structures. Biometrika, 1970, 57, 183-202. 
Jöreskog, K. G., \& Gruvaeus, G. RMFLA: A computer program for restricted maximum likelihood factor analysis (Research Bulletin 67-21). Princeton NJ: Educational Testing Service, 1967.

Jöreskog, K. G., \& Sörbom, D. LISREL: Analysis of linear structural relationships by the method of maximum likelihood. User's guide. Chicago: National Educational Resources, 1978.

Kristof, W. The statistical theory of stepped-up reliability coefficients when a test has been divided into several equivalent parts. Psychometrika, 1963, 28, 221-238.

Kristof, W. Estimates of true score and error variance for tests under various equivalence assumptions. Psychometrika, 1969, 34, 489-507.

Laughlin, J. E. Comment on "Estimating the coefficients of linear models: It don't make no nevermind." Psychological Bulletin, 1978, 85, 247-253.

Lawley, D. N., \& Maxwell, A. E. Factor analysis as a statistical method. New York: Alsevier, 1971.

Ledermann, $W$. On a shortened method or estimation of mental factors by regression. Psychometrika, 1939, $4,109-116$.

Likert, R. A technique for the measurement of attitudes. Archives of Psychology, 1932 (Whole number 140).

Lord, F. M. A significance test for the hypothesis that two variables measure the same trait except for errors of measurement. Psychometrika, 1957, 22, 207-220.

Lord, F. M. Applications of item response theory to practical testing problems. Hillsdale NJ: Erlbaum, 1980.

Lord, F. M., \& Novick, M. L. Statistical theory of mental test scores. Reading MA: Addison-Wesley, 1968.

Samejima, F. Homogenous case of the continuous response model. Psychometrika, 1973, 35, 203-219.
Sörbom, D., \& Jöreskog, K. G. COFAMM: Confirmatory factor analysis with model modification. User's guide. Chicago: National Educational Resources, 1976.

Wainer, H. Estimating coefficients in linear models: It don't make no nevermind. Psychological Bulletin, 1976, 83, 213-217.

Wainer, H. On the sensitivity of regression and regressors. Psychological Bulletin, 1978, 85, 267-273.

Weiss, David J., \& Davison, Mark L. Test theory and methods. Annual Review of Psychology, 1981, 32 , 629-658.

White, G. L. Relative involvement, inadequacy, and jealousy: A test of a causal model. Unpublished manuscript, 1979.

White, G. L. Some correlates of romantic jealousy. Journal of Personality, 1981, 49, 129-147.

\section{Acknowledgments}

This research was supported in part by a grant from the University of Kansas General Research Fund. The authors thank an anonymous reviewer who helped fill gaps in the literature review.

\section{Author's Address}

Send requests for reprints or further information to David Thissen, Department of Psychology, University of Kansas, Lawrence KS 66045, U.S.A., Thomas Pyszczynski, Department of Psychology, University of Wisconsin, Milwaukee WI 53201, or Jeff Greenberg, Department of Psychology, University of Arizona, Tucson AZ 85721. 